\title{
Procedimentos éticos de pesquisas junto a crianças, adolescentes e suas famílias em situação de violências: Um olhar necessário
}

\author{
Luiza Cesar Costa Riani'1, Fabiano Henrique Oliveira Sabino', Marília \\ Gonçalves ${ }^{2}$ e Diene Monique Carlos ${ }^{1}$ \\ 1 Departamento de Enfermagem Universidade Federal de São Carlos, Brasil | luiza- \\ riani@hotmail.com; fabianooliveira163@gmail.com; diene_enf@hotmail.com | \\ https://orcid.org/0000-0002-4950-7350; https://orcid.org/0000-0003-3182-3408; \\ https://orcid.org/0000-0001-8728-9238 \\ 2 Departamento de Psicologia Universidade Federal de São Carlos, Brasil | \\ mariliagoncalves6@gmail.com | https://orcid.org/0000-0003-4547-7235
}

Resumo: Introdução: No Brasil, crianças e adolescentes figuram como as principais vítimas de violência doméstica, sexual ou outros tipos de violência interpessoal e autoprovocada. A atualidade pluraliza as tipificações de violência, gerando margem para dubiedade em relação aos preceitos de cada cenário. Emerge-se então, diante deste cenário, a grande necessidade de compreensão, investigação e aprofundamento destes fenômenos e das populações envolvidas neles; entretanto, questões como privacidade, sigilo e segurança podem produzir dilemas éticos para pesquisadores, participantes do estudo e profissionais. Objetivo: relatar e refletir sobre os procedimentos éticos de duas pesquisas qualitativas, uma junto a famílias envolvidas em violências interpessoais contra crianças e adolescentes e outra junto a adolescentes envolvidos em violências autoinfligidas. Métodos: relato de experiência de dois estudos qualitativos realizados em municípios de médio porte do estado de São Paulo, Brasil. Resultados: A adoção de abordagens metodológicas que favoreçam a escuta ampliada e o acolhimento dos participantes, para além da coleta de informações, deve ser priorizada; ademais das estratégias de cuidado continuado, como encaminhamento para serviços de saúde e articulação com o território. Os campos de estudo precisam ser parceiros e efetivamente coparticipantes desta construção que envolve pesquisas e cuidados em saúde que se tornam indissociáveis, e não simplesmente cenários de pesquisa. Conclusões: $\mathrm{O}$ debate durante a formação dos profissionais que lidam com os casos das tipificações de violência deve perpassar os muros da academia e da prática, além disso, pesquisas sobre violências envolvendo crianças, adolescentes e suas famílias são desafiadoras do ponto de vista ético, legal, técnico e econômico. Entretanto, tais populações devem ser vistas e ouvidas na construção de conhecimento científico, principalmente em assuntos que os envolvem diretamente.

Palavras-chave: Criança; Adolescente; Violência; Ética.

Ethical Research Procedures with Children, Adolescents and Their Families in Situations of Violence: A Necessary Look

Abstract: Introduction: In Brazil, children and adolescents are the main victims of domestic, sexual and other types of interpersonal and self-harm. Nowadays pluralizes the typifications of violence, generating room for dubiousness in relation to the precepts of each scenario. Then, in view of this scenario, the great need to understand, investigate and deepen these phenomena and the populations involved in them emerges; however, issues such as privacy, secrecy and security can produce ethical dilemmas for researchers, study participants and professionals. Objective: to report ethical research procedures with families involved in interpersonal violence against children and adolescents and with adolescents involved in self-inflicted violence. Methods: experience report of two studies carried out in medium-sized municipalities in the state of São Paulo, Brazil. Results: The adoption of methodological approaches that favor extended listening and welcoming the participants, in addition to the collection of information, should be prioritized; in addition to continuing care strategies, such as referral to health services and articulation with the territory. The fields of study need to be partners and effectively co-participants in this construction of knowledge, and not simply research scenarios. Conclusions: The debate during the training of professionals who deal with cases of types of violence must pass through the walls of academia and practice, in addition, research on violence involving children, adolescents and their families is challenging from an ethical, legal point of view, technical and economical. However, such populations must be seen and heard in the construction of scientific knowledge, especially in matters that directly involve them.

Keywords: Child; Adolescent; Violence; Ethics. 


\section{Introdução}

A violência é considerada um grave problema de saúde pública; algumas populações, como crianças e adolescentes, se apresentam mais vulneráveis a este fenômeno, em especial pelo particular momento de desenvolvimento socioemocional e cognitivo. Tais situações implicam no risco de lesões e adoecimentos físicos, bem como provável presença de sofrimento psíquico, atraso no desenvolvimento, pensamentos e comportamentos suicidas (Lloyd \& Richardson et al, 2015; World Health Association (WHO, 2014).

A violência é entendida como o uso intencional da força ou do poder físico, real ou em ameaça, contra uma pessoa, grupo ou comunidade, que resulte ou tenha grande possibilidade de resultar em injúria, morte, dano psicológico, privação ou alteração de desenvolvimento (Vpa, 2014). A Organização Mundial da Saúde (OMS) identifica três tipologias de violência, de acordo com a relação entre vítima e autor da violência, a saber: violência autoprovocada; coletiva e interpessoal (WHO, 2014).

No Brasil, crianças e adolescentes figuram como as principais vítimas de violência doméstica, sexual ou outros tipos de violência interpessoal, constituindo $44,7 \%$ do total de casos notificados (Brasil, 2013). A violência autoprovocada engloba comportamentos suicidas e autolesões. No mundo, a primeira causa de morte entre jovens de 15 a 29 anos é a violência interpessoal, e a segunda é o suicídio (WHO, 2018). Já no Brasil, em 2015, o suicídio foi a quarta causa de morte nesta faixa etária, segundo dados do Ministério da Saúde (Brasil, 2017).

Há grande necessidade de compreensão, investigação e aprofundamento destes fenômenos e das populações envolvidas neles; entretanto, questões como privacidade, sigilo e segurança podem produzir dilemas éticos para pesquisadores, participantes do estudo e profissionais. Em âmbito internacional, a Organização das Nações Unidas para a Educação, a Ciência e a Cultura (UNESCO) produziu a Declaração Universal sobre Bioética e Direitos Humanos, que é um guia para decisões e práticas de indivíduos, grupos, comunidades, instituições e empresas públicas e privadas. Este documento retrata a importância da incorporação de princípios éticos do respeito à dignidade humana em todas as práticas, incluindo investigações científicas (UNESCO, 2005). Já no Brasil, a Resolução no 510 de 07 de abril de 2016 regulamenta normas aplicáveis a pesquisas com seres humanos nas Ciências Humanas e Sociais; foi entendida como um grande avanço pelas particularidades dos estudos nesta área. É aplicável a todos procedimentos metodológicos que envolvam o uso de dados diretos obtidos com participantes ou informações identificáveis. Esta resolução traz princípios éticos essenciais a serem considerados, a saber, a liberdade; autonomia; valores culturais, sociais, morais e religiosos; não preconceito e estigmatização dos participantes. Ressalta cuidados pelos pesquisadores como recusa do autoritarismo; garantia do livre assentimento e consentimento e da confidencialidade das informações e privacidade dos participantes; compromisso de assistência a danos materiais e imateriais eventuais, de forma contínua e oportuna. Finalmente, ressalta a importância da devolutiva e socialização do conhecimento produzido, de forma acessível aos participantes (Brasil, 2016).

Compreendendo a importância de refletir sobre estes aspectos, este artigo tem como objetivo relatar e refletir sobre os procedimentos éticos de duas pesquisas qualitativas, uma junto a famílias envolvidas em violências interpessoais contra crianças e adolescentes e outra, junto a adolescentes envolvidos em violências autoinfligidas. 


\section{Relatos de Experiência}

Ao longo deste item, apresentaremos relatos de experiência de dois estudos qualitativos para discussão e reflexão dos cuidados éticos em pesquisas junto a crianças, adolescentes e suas famílias em situação de violências. Pesquisas com relatos de experiência utilizam-se de uma ferramenta da pesquisa qualitativa descritiva que possibilita reflexão sobre uma ação ou situação vivenciada no âmbito profissional das autoras e de interesse da comunidade científica (Daltro \& De Faria, 2019). O relato de experiência não permite e nem objetiva se estabelecer como universal e replicável, pois se trata de uma construção teórico-prática de um acontecido, a partir do olhar do sujeito pesquisador, que localiza a experiência em seu contexto em busca de construir reflexões teoricamente embasadas e saberes inovadores (Daltro \& De Faria, 2019).

$\mathrm{O}$ primeiro estudo relatado teve por objetivo compreender os significados atribuídos à negligência contra crianças sob a ótica de famílias envolvidas neste fenômeno. O campo de estudo foi um município de médio porte do interior do estado de São Paulo. Os participantes foram os/as autores/as da negligência contra crianças, notificadas junto ao Conselho Tutelar (CT) do município. Pelas especificidades do contexto e dos participantes, reforça-se que foram discutidas e estabelecidas junto ao campo as estratégias para aproximação e garantia da continuidade do cuidado às famílias participantes.

A coleta de dados ocorreu entre os meses de dezembro e janeiro de 2021. Um contato prévio foi realizado com os participantes pelo CT de modo a alertá-los sobre a realização da pesquisa e que um pesquisador entraria em contato posteriormente. Sete dias antes da realização da entrevista o pesquisador fez um novo contato afim de fortalecer a data e horário que o sujeito foi convidado a estar no serviço de referência. Ressalta-se que em nenhum momento foi pontuado aos participantes que seriam convidados a participar da pesquisa por serem suspeitos de negligência contra crianças e adolescentes, mas apenas por estarem em acompanhamento no CT. Tal prerrogativa é expressa nas resoluções éticas como "motivo encoberto".

Obtendo o aceite, o participante realizou uma entrevista individual de duração aproximada de 30 minutos, para discussão do que seria cuidado e negligência de crianças; e os motivos da ocorrência de negligência nas famílias. É importante ressaltar que antes do início da coleta de dados, foi realizada uma ambientação, ou rapport, para ambientação e aquecimento dos participantes, antes de perguntas mais sensíveis. Assim, inicialmente foi realizado a caracterização socioeconômica. Também optou-se por iniciar com um questionamento sobre cuidado, para posteriormente adentrar possíveis elementos da negligência. Foi necessário apenas um encontro com vinte participantes. Estes foram contactados primeiramente pelo CT, a partir dos dados de notificação do fenômeno em estudo, nos quais foram preservados todos os aspectos éticos para que não se sentissem intimidados ou acusados de tal violação de direitos, sendo caracterizados por números à medida que as entrevistas foram realizadas.

Durante todo o processo, desde a entrada do entrevistado no CT até a sua saída, foi reforçada a importância dele para a pesquisa. Tal ato empoderou o participante e o encorajou a relatar suas percepções e experiências sobre o tema. Foi ressaltado que, apesar da pesquisa ter sido realizada no setor de referência, dentro da sala não haveriam espaços para julgamentos pessoais e nem interpretações subjetivas. O papel do pesquisador era apenas ouvir os relatos dos participantes de modo a não se sentirem diminuídos, taxados, julgados ou menosprezados.

Para tal, caso o participante sentisse incômodo, tristeza ou qualquer outro sentimento negativo, com alguma pergunta ou durante as discussões, ou por constrangimento com alguma informação concedida, ele (a) poderia optar pelo encerramento da entrevista e o pesquisador se ofereceria a auxiliar o entrevistado a encontrar um serviço de atendimento continuado. 
Quanto aos custos, foi reforçado que a pesquisa não seria de natureza remunerativa para o participante ou para o pesquisador, porém, caso houvesse custos de qualquer natureza, seriam reembolsados pela equipe de pesquisa. Foi estimulado ainda, que os participantes buscassem um horário melhor adequasse à rotina cotidiana para participar do estudo.

Após as entrevistas, a pesquisa foi finalizada com o Mapa Mínimo da Rede Pessoal Social (Sluzki, 1997). Este é um instrumento de fácil entendimento e baixo custo que permite ao participante refletir e falar sobre suas relações pessoais e institucionais, preenchendo um mapa de acordo com a natureza, qualidade e a intensidade destas relações. O preenchimento do mapa possibilitou um fechamento das conversas de forma leve e acolhedora, além de servir como instrumento terapêutico por identificar possibilidades de apoio social. Neste aspecto, é importante reforçar o preparo técnico dos pesquisadores para oferecer este cuidado.

O segundo estudo teve por objetivo identificar e analisar as concepções de autolesão não suicida (ALNS) por adolescentes que se autolesionam. O interesse em estudar a violência autoprovocada na adolescência surgiu durante a realização de atividades de promoção de saúde em escolas da rede pública de uma cidade do interior de São Paulo, quando a problemática da ALNS e dos comportamentos suicidas mostrou-se presente e recorrente, tanto nos discursos dos adolescentes, quanto como preocupação por parte dos profissionais de educação. O estudo foi realizado em uma destas escolas da rede pública da cidade.

$\mathrm{Na}$ unidade de realização do estudo, a coordenação identificou entre os estudantes que haviam adolescentes com histórico de ALNS. A partir desta identificação, a escola acolheu os adolescentes e comunicou as famílias, antes do início da pesquisa. Após a elaboração do projeto de pesquisa, o serviço informou às famílias sobre a existência do estudo, e pediu consentimento para a participação dos adolescentes, pela assinatura do Termo de Consentimento Livre e Esclarecido. Após a ciência dos pais, as adolescentes foram convidadas a participar da pesquisa, e assinaram o Termo de Assentimento Livre e Esclarecido (TALE). Vale ressaltar que, antes do início da coleta de dados, as pesquisadoras conversaram com os familiares das adolescentes e ofertaram escuta, acolhimento e esclarecimento de eventuais dúvidas.

A preocupação e reflexão sobre os aspectos éticos da pesquisa pautaram a construção da estratégia metodológica, recrutamento de participantes, execução e divulgação de dados da pesquisa. A estratégia de coleta de dados foi o uso da Consulta Terapêutica individual, mediada pelo Procedimento Desenho-Estória com Tema, com o tema Automutilação. Consulta terapêutica é uma técnica desenvolvida pelo psicanalista Winnicott, que consiste em poucos encontros (hum a três), conta com o uso de um mediador dialógico para expressão e privilegia o acolhimento dos conteúdos ideoafetivos emergentes (Winnicott, 1984). Entendeu-se ser adequada ao estudo por permitir, ao mesmo tempo, coletar dados importantes para uma investigação e fazer intervenção em conteúdos que causem sofrimento psicossocial ao participante. A escolha desta metodologia se respaldou nos cuidados éticos e trouxe benefícios para ambas, investigadora e participante. Ainda pensando em acessar as vivências destas adolescentes, optou-se pela utilização do Procedimento Desenho-Estória com Tema de Aiello-Vaisberg (1999), que consistiu em solicitar à participante que fizesse um desenho com o tema "Automutilação" e contasse uma história sobre sua produção gráfica. A pesquisadora realizava os mesmos passos que a participante. A escolha deste procedimento deveu-se à sua caraterística de facilitar a imersão de conteúdos de difícil expressão oral, considerando que a literatura relaciona a autolesão não suicida com a dificuldade de expressividade e comunicação (Aiello-Vaisberg,1999).

No último encontro com cada adolescente, também foi realizada a construção do Mapa Mínimo da Rede Pessoal Social (Sluzki, 1997). Ressalta-se que, quando identificada a necessidade, as participantes foram encaminhadas para serviços de saúde e assistência social. 
Foi garantido a todas as participantes e suas famílias o acesso a serviços de saúde que pudessem oferecer cuidado longitudinal, sendo que o projeto de pesquisa foi discutido com a rede de saúde do território antes de sua realização. Para além de todo cuidado ético na escolha do percurso metodológico, com um instrumento que também permite uma elaboração do vivido por parte das participantes, as pesquisadoras participavam do convívio escolar por meio de um projeto de extensão com o objetivo de promover saúde física e mental a partir de abordagens participativas junto à comunidade. Tal aspecto facilitou a aproximação e continuidade do olhar aos adolescentes.

No atual cenário de emergência sanitária, devem ser tomadas medidas adicionais para garantir a segurança dos pesquisadores e dos participantes. O primeiro estudo aqui descrito foi realizado durante o período da pandemia, e respeitaram-se todos os protocolos de segurança presentes a partir da pandemia de COVID-19 por meio da construção de um plano de contingência. Houve distanciamento de dois metros entre 0 entrevistado e o entrevistador, em ambiente arejado com circulação ampla de ar. Foi ofertado a todos os participantes álcool em gel a concentração $70 \%$ e, a cada finalização de entrevista, o espaço era higienizado com álcool $70 \%$ líquido, incluindo cadeira, mesa, maçanetas e todos os objetos tocados pelos presentes na sala. Todos os presentes estavam de máscara e se mostraram cientes e praticantes das medidas de distanciamento e etiqueta respiratória. A segurança do participante também é responsabilidade do pesquisador e um cuidado ético. Entende-se que tais fenômenos não poderiam ser acessados, discutidos e refletidos por meio de tecnologias virtuais e remotas; pela sensibilidade dos temas, a atual situação de pandemia apresenta-se como desafio a ser enfrentado contextualmente.

\section{Discussão}

A reflexão acerca dos princípios éticos em pesquisa, dos direitos e da proteção dos participantes não esteve sempre em pauta. Observamos ao longo da história diversos estudos que desrespeitaram os direitos fundamentais dos participantes, e que, em geral, não consideravam a participação de crianças e adolescentes (Kipper, 2016). Tais discussões puderam se iniciar internacionalmente a partir do Código de Nüremberg, que introduziu o conceito de autonomia dos participantes, e do dever dos pesquisadores quanto ao cumprimento dos seus direitos, (Organização das Nações Unidas [ONU], 1949). Outro passo importante em direção à ética em pesquisa com crianças e adolescentes foi a Declaração de Helsinki, redigida em 1964, que prevê a participação de pessoas com menos de 18 anos em estudos científicos, desde que haja consentimento de seu responsável legal, e mais recentemente, do assentimento da própria criança/adolescente, respeitando a compreensão dos participantes (World Medical Association [WMA], 2009).

Por vezes, o conhecimento das questões éticas perpassa pela subjetividade da experiência profissional, o que de fato é importante, mas não ultrapassa os limites do conhecimento tácito. A aplicação da ética na pesquisa deve ser pautada na compreensão do fenômeno como um todo, de forma que possa compreender que muitas vezes o comportamento que infere na violência ou na violação de direitos é resquício ou reflexo de um comportamento social e cultural (Garcia \& Beiras, 2019).

Pesquisas com crianças e adolescentes podem ser mais desafiadoras do que pesquisas com adultos, sobretudo quando o objeto de estudo é a violência. Os desafios incluem aspectos éticos, legais, técnicos e econômicos. Entretanto, mesmo diante das dificuldades, essas pesquisas são necessárias e possíveis, e vêm sendo desenvolvidas em todo o mundo (Kipper, 2016). As crianças, os adolescentes e suas famílias devem ser vistos e ouvidos na construção de conhecimento científico, principalmente em assuntos que os envolvem diretamente, como é o caso das violências.

As pesquisas sobre violência com crianças, adolescentes e suas famílias devem visar também o benefício dos participantes e de seu entorno, para além da obtenção de dados científicos. 
Neste sentido, na construção do percurso metodológico, ambos os estudos utilizaram-se de estratégias que favorecessem o diálogo e a construção de um espaço acolhedor e livre de julgamentos. Ao finalizar a coleta de dados, os pesquisadores convidaram os participantes a construir o Mapa Mínimo da Rede Pessoal Social, como forma de oferecer também aos participantes uma visão organizada de sua rede de suporte (Carlos et al., 2019).

Protocolos de estudos devem obrigatoriamente buscar minimizar riscos e danos aos participantes. Em recente revisão sistemática de estudos indianos para compreender práticas e lacunas éticas em estudos sobre violência sexual infantil encontrou-se que apenas $25 \%$ dos estudos discutiam tais métodos. A maioria deles se referiu ao aconselhamento de serviços aos participantes quando necessário e o referenciamento a serviços psiquiátricos ou gerais (Dayal et al, 2018). Em estudos junto às famílias envolvidas em situações de violências é muito importante o cuidado para não estigmatização e descriminação rotulagem.

No caso do primeiro estudo, debater questões sobre a negligência familiar possibilita o esclarecimento de manifestações e discursos culturais que transcendem a lógica da responsabilização meramente punitiva executada junto às famílias. As políticas públicas são ferramentas importantes na construção da saúde familiar, porém a família não é parte menos importante neste contexto e nem indissociável a ele, necessitando ser ouvidas e ativas nesta construção (Egry et al, 2015).

$\mathrm{Na}$ escolha do percurso metodológico, optou-se por não aplicar instrumentos ou questionários nos adolescentes para medir a prevalência ou grau da violência autoprovocada, pois foi demonstrado em estudo que se debruçou sobre as recomendações éticas para estudos com adolescentes que se autolesionavam que estes instrumentos específicos e detalhados podem despertar a ideia de se automutilar ou ensinar novos métodos para pessoas que não os conheciam anteriormente. $O$ mais recomendado eticamente é que os pesquisadores perguntem se o adolescente já se autolesionou, em lugar de aplicar instrumentos para identificá-los (Hasking et al., 2019).

A literatura demonstra que adolescentes que participam em pesquisa sobre violência autoprovocada relatam que a experiência proporciona maior entendimento sobre si mesmos e sobre maneiras saudáveis de enfrentar o sofrimento (Jorm et al., 2007; Hasking et al, 2015). Hasking et al (2019) aponta, ainda, que há evidências crescentes de que perguntar sobre a violência autoprovocada para adolescentes não aumenta a angústia ou o risco de se envolver nela. Em vez disso, em estudos com adolescentes que se autolesionavam, os participantes perceberam benefícios para si mesmos da participação de pesquisas sobre o tema, e que sua participação os alertou sobre a ajuda disponível. Finalmente, aponta-se para a necessidade do desenvolvimento de pesquisas que oferecem espaço de expressão para adolescentes e que lancem luz sobre a experiência subjetiva destes sujeitos, sendo que permitir que 0 discurso dos adolescentes seja ouvido tem uma dimensão ético-política de construção de saber e possibilidade promoção de saúde e bem-estar (Oliveira et al, 2012).

\section{Considerações Finais}

Esta reflexão trouxe que pensar e realizar investigações que se propõem a ouvir crianças, adolescentes e suas famílias envolvidos em situações de violências demandam reflexões e cuidados pautados nos princípios éticos e nas regulamentações vigentes. $O$ estudo emergiu a importância da pesquisa qualitativa em quaisquer temas que exijam um aprofundamento das experiências, vivências e significados, inclusive na redução de danos frente a fenômenos sensíveis, mas que têm necessidade de serem melhor pesquisados. Neste aspecto, tem ainda um imperativo ético, humanístico e social ao ouvir as vozes dos diretamente envolvidos no fenômeno. 
As medidas restritivas relacionadas à pandemia devem respeitar os preceitos de segurança sanitária aliados à ética. As ações para minimizar os riscos durante a participação devem ser pensadas com responsabilidade e rigor, principalmente por se tratar de uma população vulnerável e de temas que podem gerar desconforto ou sofrimento. A adoção de abordagens metodológicas que favoreçam a escuta ampliada e - acolhimento dos participantes, para além da coleta de informações, deve ser priorizada; ademais as estratégias de cuidado continuado, como encaminhamento para serviços de saúde e articulação com o território, devem ser vislumbradas. Reforça-se que o experienciamento das violências requer uma visão caleidoscópica dos fenômenos, reforçando a complexidade humana na construção social. Os campos de estudo precisam ser parceiros e efetivamente coparticipantes desta construção de conhecimento, e não simplesmente cenários de pesquisa.

A aglutinação entre os relatos das pesquisas permitiu evidenciar que, tanto pesquisas com vítimas quanto com autores da violência exige do pesquisador uma visão ampla e despida de subjetividades e julgamentos pessoais. Finalmente, o estudo traz implicações para pesquisas junto a populações envolvidas nas violências, bem como para a gestão e produção de conhecimento na área.

\section{Referências}

Aiello-Vaisberg, T. M. J. (1999). Encontro com a loucura: transicionalidade e ensino de psicopatologia (Tese de Doutorado, Universidade de São Paulo).

Brasil. Conselho Nacional de Saúde (2013). Resolução no 466, de 12 de dezembro de 2012. Resolve aprovar as seguintes diretrizes e normas regulamentadoras de pesquisas envolvendo seres humanos. Brasília : Diário Oficial da União., 59, 13 jun 2013. Seção 1.

Brasil. Ministério da Saúde. (2017). Secretaria de Vigilância em Saúde. Departamento de Vigilância de Doenças e Agravos Não Transmissíveis e Promoção da Saúde. Viva : Vigilância de Violências e Acidentes : 2013 e 2014 [recurso eletrônico] / Ministério da Saúde, Secretaria de Vigilância em Saúde, Departamento de Vigilância de Doenças e Agravos Não Transmissíveis e Promoção da Saúde. - Brasília: Ministério da Saúde.

Carlos, D. M., Pereira, S. L. M., Beserra, M. A., Aragao, A. D. S., Gregory, A., Carvalho, F, M, D, G. (2019). Social support network of family members of abused children and adolescents: Perspectives and possibilities. Journal Of Clinical Nursing, 28( 5-6), 814-827.

Daltro, M. R., de Faria, A. A. (2019). Relato de experiência: Uma narrativa científica na pósmodernidade. Estudos e pesquisas em psicologia, 19(1), 223-237.

Dayal, R., Kalokhe, A.S., Choudhry, V. et al. (2018). Ethical and definitional considerations in research on child sexual violence in India. BMC Public Health, 18, 1144. https://doi.org/10.1186/s12889-018-6036-y

Egry, E. Y. et al. (2015). Compreendendo a negligência infantil na perspectiva de gênero: estudo em um município brasileiro. Rev Esc Enferm USP. 49(4),556-563. DOI: 10.1590/S0080 623420150000400004

Egry, E. Y., Apostolico, M. R., \& Morais, T. C. P. (2018). Notificação da violência infantil, fluxos de atenção e processo de trabalho dos profissionais da Atenção Primária em Saúde. Ciência \& Saúde Coletiva, 23, 83-92.

Garcia, A. L. C., \& Beiras, A. (2019). A psicologia social no estudo de justificativas e narrativas de homens autores de violência. Psicologia: Ciência e Profissão, 39(SPE2).

Hasking, P., Tatnell, R. C., \& Martin, G. (2015). Adolescents' reactions to participating in ethically sensitive research: a prospective self-report study. Child and adolescent psychiatry and mental health, 9(1), 1-12.

Hasking, P. A., Lewis, S. P., Robinson, K., Heath, N. L., \& Wilson, M. S. (2019). Conducting research on nonsuicidal self-injury in schools: Ethical considerations and recommendations. School psychology international, 40(3), 217-234.

Jorm, A. F., Kelly, C. M., \& Morgan, A. J. (2007). Participant distress in psychiatric research: A systematic review. Psychological medicine, 37(7), 917. 
Kipper, D. J. (2016). Ética em pesquisa com crianças e adolescentes: à procura de normas e diretrizes virtuosas. Revista Bioética, 24(1), 37-48.

Kitano, N., Lee, K., \& Nakamura, Y. (2019). Demographic characteristics of foreign residents in Japan; child maltreatment and language problems in child protection services. Nihon eiseigaku zasshi. Japanese journal of hygiene, 74

Konijnendijk, A. A., Boere-Boonekamp, M. M., Haasnoot, M. E., \& Need, A. (2019). Effects of a computerised guideline support tool on child healthcare professionals' response to suspicions of child abuse and neglect: a community-based intervention trial. BMC medical informatics and decision making, 19(1), 1-12.

Lescovar, G. Z. (2004). As consultas terapêuticas e a psicanálise de DW Winnicott. Estudos de Psicologia (Campinas), 21(2), 43-61.

Oliveira, T. G. P. D., Muylaert, C. J., \& Reis, A. O. A. (2012). Crianças como sujeitos na pesquisa: uma revisão integrativa. Psicologia Hospitalar, 10(2), 02-16.

Okato, A., Hashimoto, T., Tanaka, M., Tachibana, M., Machizawa, A., Okayama, J., ... \& lyo, M. (2018). Hospital-based child protection teams that care for parents who abuse or neglect their children recognize the need for multidisciplinary collaborative practice involving perinatal care and mental health professionals: a questionnaire survey conducted in Japan. Journal of multidisciplinary healthcare, $11,121$.

Organização das Nações Unidas (ONU) (1949). Código de Nüremberg. Disponível: http://www.ufrgs.br/bioetica/nuremcod.htm

Organização das Nações Unidas para a Educação, a Ciência e a Cultura (UNESCO) (2005). Declaração Universal sobre Bioética e Direitos Humanos. Paris(FR): UNESCO. Disponível em: https://unesdoc.unesco.org/images/0014/001461/146180por.pdf.

Pasian, M. S., Faleiros, J. M., Bazon, M. R., \& Lacharité, C. (2013). Negligência infantil: a modalidade mais recorrente de maus-tratos. Pensando famílias, 17(2), 61-70.

Violence Prevention Alliance (VPA) (2014). Definition and typology of violence. Geneva: World Health Organization.

World Health Organization. (2019). The WHO special initiative for mental health ( 2019-2023): universal health coverage for mental health (No. WHO/MSD/19.1). World Health Organization.

Winnicott, D. W. (1983). O ambiente e os processos de maturação: estudos sobre a teoria do desenvolvimento emocional. Artes Médicas.

Winnicott, D. W. (1984). Consultas terapêuticas em psiquitria infantil. Rio de janeiro: Imago.

World Medical Association. (2009). Declaration of Helsinki. Ethical principles for medical research involving human subjects. Jahrbuch für Wissenschaft und Ethik, 14(1), 233-238. 